\title{
Preliminary Evaluation of Automated Speech Recognition Apps for the Hearing Impaired and Deaf
}

1 Leontien Pragt ${ }^{1 *}$, Peter van Hengel ${ }^{1,2}$, Dagmar Grob ${ }^{3}$, Jan-Willem Wasmann ${ }^{1}$

$2{ }^{1}$ Department of Otorhinolaryngology, Donders Institute for Brain, Cognition and Behaviour,

3 Radboud University Medical Center Nijmegen, the Netherlands;

$4 \quad{ }^{2}$ Pento Audiological Center Twente, Hengelo, The Netherlands;

$5 \quad{ }^{3}$ Department of Medical Imaging, Radboud University Medical Center, Nijmegen, The Netherlands.

6 * Correspondence:

7 Corresponding Author

8 Leontien.Pragt@radboudumc.nl

9 Keywords: ASR, Automated Speech Recognition, Automatic Speech Recognition, Automated

10 speech audiometry, Evaluation Metric, Speech Reception, Speech-to-Text, Hearing

11 Impairment, Voice-to-Text Technology

13 ABSTRACT

14 Objective. Automated speech recognition (ASR) systems have become increasingly sophisticated,

15 accurate, and deployable on many digital devices, including on a smartphone. This pilot study aims

16 to examine the speech recognition performance of ASR apps using audiological speech tests. In

17 addition, we compare ASR speech recognition performance to normal hearing and hearing impaired 
18 listeners and evaluate if standard clinical audiological tests are a meaningful and quick measure of

19 the performance of ASR apps.

20 Methods. Four apps have been tested on a smartphone, respectively AVA (iOS, Android), Earfy

21 (iOS, Android), Live Transcribe (Android) and Speechy (iOS). The Dutch audiological speech tests

22 performed were speech audiometry in quiet (Dutch CNC-test), Digits-in-Noise (DIN)-test with

23 steady-state speech-shaped noise, sentences in quiet and in averaged long-term speech-shaped

24 spectrum noise (Plomp-test). For comparison, the apps' ability to transcribe a spoken dialogue

25 (Dutch and English) was tested.

26 Results. All apps scored at least 50\% phonemes correct on the Dutch CNC-test for a conversational 27 speech intensity level (65 dB SPL) and achieved 90-100\% phoneme recognition at higher intensity

28 levels. On the DIN-test, AVA (iOS, Android) and Live Transcribe (Android) had the lowest (best)

29 signal-to-noise ratio $+8 \mathrm{~dB}$. The lowest signal-to-noise measured with the Plomp-test was +8 to $9 \mathrm{~dB}$

30 for Earfy (Android) and Live Transcribe (Android). Overall, the word error rate for the dialogue in

31 English (19-34\%) was lower (better) than for the Dutch dialogue (25-66\%).

32 Conclusion. The performance of the apps was limited on audiological tests that provide little

33 linguistic context or use low signal to noise levels. For Dutch audiological speech tests in quiet, ASR

34 apps performed similarly to a person with a moderate hearing loss. In noise, the ASR apps performed

35 more poorly than most profoundly deaf people using a hearing aid or cochlear implant. Adding new

36 performance metrics including the semantic difference as a function of SNR and reverberation time

37 could help to monitor and further improve ASR performance.

391 INTRODUCTION 
40 Automated Speech Recognition (ASR) has become increasingly sophisticated and accurate as a result

41 of advances in deep learning, cloud computing, and the availability of large training sets $(1,2)$. The

42 software converts speech into text using artificial intelligence models that have been trained on vast

43 collections of speech containing millions of words. ASR software is widely available on most digital

44 devices, including smartphones, tablets, or laptops. It is primarily used for voice commands (e.g. hey

45 Siri!), at the workplace to create transcripts, or in class for taking notes. Recently, ASR has become

46 available in online meetings (e.g. Microsoft teams) and video recordings (e.g. Google's Youtube) to

47 provide automatic captions. Also, several ASR-based speech-to-text apps have been developed for

48 the hearing impaired and deaf, providing live captioning of conversations $(2,3)$, showing the potential

49 of automation and artificial intelligence for hearing healthcare $(4,5)$. Early in 2020, we were

50 confronted in our clinic with questions from patients related to the use of ASR apps for daily

51 communication. These questions were especially common among patients with severe to profound

52 hearing loss who visited our outpatient clinic to assess if they were eligible for a Cochlear Implant.

53 Also, patients who had experienced sudden deafness, but had not yet been fitted with hearing aids,

54 made use of an ASR app during their appointments. There was no or little experimental information

55 at the time about the performance and usability of the ASR apps for hearing impaired persons beyond

56 what was shared by developers. Nor did we have clear criteria for which groups of patients we might

57 suggest the ASR apps to.

58 Since 2017, several ASR systems have claimed speech recognition performance close to that of

59 normally hearing humans $(1,2)$. The most common metric to express ASR performance, used to

60 underpin these claims, is the word error rate (WER). WER is calculated by adding the number of

61 missing, wrong, and inserted words and dividing this by the total number of words (6). A lower WER

62 score means better performance. The performance of ASR will be best for speech similar to the

63 speech on which it was trained (7). It is therefore important to understand for what specific task an

64 ASR is designed for and how it is evaluated. Typically ASRs are evaluated on well-studied large 
65 (>100 hours) collections of speech, referred to as a corpus. The SwitchBoard corpus and CallHome

66 corpus are well-known collections of conversational phone calls (8), whereas Librispeech is a corpus comprising speech from public domain audiobooks. The SwitchBoard corpus consists of conversations over the phone between strangers about a given topic (9). The CallHome corpus

69 consists of more informal conversations between friends and family (8). None of these corpora are ideal for use in acoustically challenging environments. The SwitchBoard and CallHome were collected under low noise and low reverberation conditions (9), and a large portion of the Librispeech corpus has undergone noise removal and volume normalization (10).

In order to obtain estimates of human speech recognition performance that could be used for comparison with ASR, some researchers have determined the WER among professional transcribers of speech from the SwitchBoard and CallHome corpora. Saon et al. (2017) estimated the lowest (best) achievable WER, 5.1\% for SwitchBoard and 6.8\% for CallHome, based on the best score taken from three professional speech transcribers after a quality check by a fourth speech transcriber (1). Xiong et al. (2017) on the other hand, followed more realistic industry standard procedures, which are similar to how speech is processed by ASR (2). The reported WERs were 5.9\% for SwitchBoard, and $11.3 \%$ for CallHome.

81 For some commonly-used systems ASR systems, WERs of 5.1\% (Microsoft) and 5.5\% (IBM) have

82 been reported using the SwitchBoard corpus (11), which is close to the performance of normal

83 hearing professionals reported above $(1,2)$. Benchmark results of widely used ASR systems tested on

84 the same corpora are not available to our knowledge. Google reported a WER of 4.9\%, but used a

85 non-public corpus (11). Koenecke et al. (2020) compared the performance of ASR systems from

86 Amazon, Apple, Google, IBM, and Microsoft to transcribe structured interviews using two recent

87 developed corpora (CORAAL and AAVE; (7)). However, transcribing a structured interview is a

88 very different task than transcribing a conversation in real-time in acoustically challenging

89 environments. More ecologically valid tasks are needed that take account the effects of noise, 
reverberation, talker accent, and slang, for instance, to provide a realistic estimate of ASR

91 performance when used for conversations in daily life under various acoustic conditions.

92 For people with hearing impairments, there are specific user needs to consider when developing ASR

93 apps. For example, these listeners might use both speechreading (12) and text reading of the ASR

94 transcript from a screen. Speechreading conveys important nonverbal cues and nuances not included

95 in a transcript and may enhance speech-in-noise abilities (13). However, without careful design,

96 reading a transcript may interfere with someone's speechreading ability. Speaker identification cues

97 (e.g by color coding each speaker a feature in AVA (14)) may also direct the reader to the face of an

98 active talker. Other design ideas include the notification of critical environmental sounds (a feature

99 incorporated in Live Transcribe (15)), feedback to the speaker of their intelligibility of the ASR, or

100 feedback to the speaker by making the transcript readable from two sides (e.g mirrored) so that both

101 the speaker and the listener can check the results (incorporated in Earfy (16)).

102 The settings where an ASR is used may also differ between individuals with impaired or normal

103 hearing. For example, the settings where people with hearing loss use ASR may be more often in a

104 more homely atmosphere between family members that might use more colloquial language or slang.

105 That situation may be similar to closed caption for video series. The most common complaint of

106 people with hearing loss is the reduced speech perception in complex listening environments

107 including cocktail parties, restaurants, in conversations with their doctor, and family gatherings

$108(15,16)$. Adverse acoustic conditions, including low signal-to-noise, make it difficult for normal

109 hearing listeners to understand speech and make the speech incomprehensible for persons with mild

110 to profound hearing loss. Finally, the speed of translation to accommodate a fluent conversation and

111 the user interface to make it practical for older users and digitally less proficient users are factors to

112 consider.

113 A standardized task that fully captures the skills of humans to recognize speech does not yet exist, to

114 our knowledge. Such a task would need to account for factors as background noise, reverberation, 
115 accent, and speech impairment. This is needed to verify claims that ASR speech recognition

116 performance is close to humans $(1,2)$ and should be done using diverse training datasets (7).

117 This pilot study aimed to examine the speech recognition performance of ASR apps using

118 audiological speech tests. We normally administer clinical audiology tests in patients from normal

119 hearing to profound hearing loss to assess speech recognition. We tested the hypothesis that our

120 clinical tests might thus provide objective metrics for performance of ASR systems for people with

121 hearing loss, helping us to determine what range of hearing losses could benefit from ASR apps. In

122 addition, we compared ASR results to normal hearing and hearing impaired listeners and evaluated if

123 standard clinical audiological tests provide a meaningful and quick measure of the performance of

124 ASR apps.

125

1262 METHODS

127 Four different apps on two smartphones, with various operating systems, were tested on their ability

128 to transcribe speech. For this project, the iOS operating apps were tested using an iPhone 6, and for

129 the Android operating apps, a Samsung A3 was used. Both smartphone devices are widely used. We

130 decided to select inexpensive ASR apps $(<\$ 10)$ since they would be most widely used by our patients

131 while the cost for ASR apps is not reimbursed in the Netherlands. The four apps tested were Ava and

132 Earfy that both run on iOS and Android, Speechy iOS only, and Live Transcribe Android only. The

133 tested apps were chosen by searching on the Internet on November $18^{\text {th }}, 2019$, for the best-known

134 speech recognition apps for the hearing impaired and deaf as well as good reviews on the different

135 app-stores. Also, the apps needed to be suitable to convert English and Dutch speech into text and

136 inexpensive (less than $\$ 10$ for a license). 
138 The apps were evaluated in similar test conditions used to assess speech reception in human listeners

139 in Dutch Audiology Centers according to best local clinical practice. The smartphones were placed

140 one meter in front of a speaker in a sound treated room compliant with ISO 8253-1 (19). Standard

141 clinical calibration protocols were used for all speech material. The microphone of the smartphone

142 was aimed towards the speaker, which we assumed to be the optimal microphone orientation, at

143 approximately the height of a listeners' ears to resemble testing conditions when tested with human

144 listeners. The smartphone screen was facing upwards allowing the experimenter to read the text from

145 the screen. Four different speech reception tests were performed to evaluate the apps' ability to

146 convert speech into text.

148 --- insert figure 1 around here ---

150 First, the apps were tested on speech recognition in quiet by converting a list of single words into

151 text. The standard Dutch speech recognition test for this purpose was the Dutch CNC-test, which

152 consists of phonetically balanced lists of twelve monosyllabic Dutch words in quiet (CNC-list,

153 'Nederlandse Vereniging voor Audiologie'; (20)). The words were played through a speaker, scored

154 and displayed in a phoneme recognition score. All words consisted of three phonemes with a

155 consonant-nucleus-consonant (CNC) structure. The first word was a test word and was not included

156 in the scoring. A human observer performed the scoring by reading the word from the screen and

157 counting the number of correct phonemes. Inserted phonemes were subtracted from the score

158 according to the clinical scoring procedure (20). If a displayed word changed during the test, the final

159 word was scored. A 100\% phoneme recognition score was reached if all 33 phonemes of the 11

160 words were correct. Several lists were presented at an intensity level of 45, 55, 65, 75, and $85 \mathrm{~dB}$

161 sound pressure level (SPL) and the speech recognition as a function of presentation level (known in 
162 human listeners as speech audiogram) is plotted for each app. For comparison, normal hearing

163 listeners achieve 100\% phoneme recognition at $45 \mathrm{~dB}$ SPL (20).

165 Second, the Plomp-test (Dutch sentences in noise) was presented (21). The test consists of 13

166 sentences of 8 to 9 syllables presented in noise with the same averaged long-term spectrum as the

167 speech. A sentence was scored to be either correct, if the whole sentence was correctly presented on

168 the screen, or incorrect, which was according to the conventional scoring procedure in clinical

169 practice (22). The speech recognition threshold (SRT) in noise was defined as the signal-to-noise

170 ratio (SNR) expressed in $\mathrm{dB}$ where on average $50 \%$ of the time the sentences were transcribed

171 correctly, following the adaptive procedure described by Plomp and Mimpen (1979; 20). The test

172 was first performed without the noise to obtain the SRT in quiet. Afterward, the masking noise level

173 was set 15-20 dB above the SRT of the apps in the quiet situation, which was $70 \mathrm{~dB}$ SPL for all apps,

174 to determine the speech reception threshold (SRT) in noise.

176 Third, a DIN-test (Digits-in-Noise) was performed. Digit triplets (e.g. 125 ) were presented in a

177 long-term average speech-spectrum noise via a 1-up, 1-down adaptive SNR procedure. SRT was

178 expressed in $\mathrm{dB}$ SNR, where a listener can on average recognize $50 \%$ of the digit triplets correctly. A

179 test series consisted of 24 triplets. The first four triples were not used to determine the test outcome.

180 The noise level was set at a fixed level of $60 \mathrm{~dB}$ with an initial positive SNR of $6 \mathrm{~dB}$. The stepsize to

181 adjust the level of the triplets was $2 \mathrm{~dB}$. The DIN-test has a measurement error in humans of $0.7 \mathrm{~dB}$

182 (23).

183

184 Finally, a fragment of dialogue in Dutch and English at $72.2 \mathrm{~dB}(\mathrm{~A})$ was presented through the

185 speaker to recreate a more realistic listening setting. The Dutch dialogue was an introduction video of

186 the Radboudumc with a female voice, talking clearly and at a normal pace 
187 (https://www.youtube.com/watch?v=zBJBD1-ePRw). For the English dialogue, part of an advanced

188 English tutorial was played. In this video, a conversation could be heard between a male and female 189 voice (https://www.youtube.com/watch?v=JtMgw2rCYSo\&t=1s). The Dutch dialogue consisted of

190256 words, while the English dialogue consisted of 248 words. After the whole dialogue was played,

191 scoring was performed on the transcript outputted by the app. The number of missing, wrong, and

192 inserted words was counted and expressed in the WER.

194 In the end, a test-retest was performed to provide insight into the accuracy of the apps. All apps were 195 retested on the $\mathrm{CNC}$-test. The test-retest reliability on the $\mathrm{CNC}$-test was visually assessed using a 196 Bland-Altman graph. The best scoring app on the DIN- and Plomp-test, one for iOS and one for 197 Android, was retested for both speech-in-noise tests. The root mean square difference (RMSD) was 198 calculated for these results. No retest was performed for the dialogue.

\section{RESULTS}

201 The results for all apps on the Dutch CNC-test (words in quiet) are shown in Figure 2. The Speech

202 Recognition as a function of presentation level was determined per app by interpolating a line using

203 logistic regression on all available-data points (test and retest measurements). A 100\% phoneme

204 recognition was reached around $80 \mathrm{~dB}$ SPL for all apps except Earfy. Earfy (iOS and Android)

205 scored 90\% words correctly around $90 \mathrm{~dB}$ SPL. The shape of the apps' "speech audiogram" curves

206 look similar to the s-shaped psychometric curve of normal hearing listeners determined by

207 Bronkhorst et al. (1993) in 20 normal hearing university students (24). However, all apps' SRT were

208 between 50 and $60 \mathrm{~dB}$ SPL, which is 25 to $35 \mathrm{~dB}$ poorer than normal hearing listeners who have a

209 SRT around $25 \mathrm{~dB}$ SPL (20). 
211 --- insert figure 2 around here ---

212

213 The speech-in-noise results are shown in Figures 3 and 4. All apps score a signal-to-noise ratio

214 (SNR) higher than $+8 \mathrm{~dB}$ on the DIN- and Plomp-test. Live transcribe (Android), and AVA

215 (Android, iOS) achieved the best results on the DIN-test. Earfy on Android performed better than on

216 iOS. Live Transcribe (Android) and AVA (iOS) achieved the best result using the Plomp-test. There

217 was a notable difference between the operating systems for AVA and Earfy when measured with the

218 Plomp-test.

219 --- insert figure $3 \& 4$ around here ---

221 In figure 5, the WER scores for both the Dutch and English dialogue are shown. Overall, the dialogue

222 in English (WER 19-34\%) was more correctly converted into words than the Dutch (WER 25-66\%)

223 dialogue. Speechy (iOS) had best matching result for English and Dutch (WER of 19\% and 20\%).

224 Earfy (iOS) showed the greatest difference between English and Dutch (WER of 19\% and 66\%).

225 --- insert figure 5 around here ---

226 The test-retest reliability of the CNC-test can be seen in figure 6. Visual inspection of the Bland-

227 Altman plot for the CNC-test-test did not show signs of any systematic bias in the relationships

228 between differences and averages. The test-retest comparison of the CNC-test showed three outliers.

229 Earfy for iOS exhibited large differences between the measurements at 70 and $90 \mathrm{~dB}$ and Live

230 transcribe (Android) had a large difference between measurements at $50 \mathrm{~dB}$. The test-retest reliability

231 on the DIN- and Plomp-tests was assessed for one Android and one iOS app. The test-retest

232 difference expressed in Root-Mean-Square-Difference on the DIN-test was $0.4 \mathrm{~dB}$ iOS Ava and 0.8 
233 dB Android Live Transcribe, which we regard as acceptable since in normal hearing listeners tested

234 monaurally using headphones, $90 \%$ of measurements are within $1.4 \mathrm{~dB}$ (measurement error is 0.70

$235 \mathrm{~dB}$ ) for a single list on the DIN-test (23). The Root-Mean-Square-Difference on the Plomp-test was

$236 \quad 0.6 \mathrm{~dB}$ iOS Ava and $2.0 \mathrm{~dB}$ for Android Live Transcribe.

237 --- insert figure 6 around here ---

238

2394 DISCUSSION

240 Main results

241 None of the ASR apps achieved performance close to normal hearing listeners on audiological tests.

242 In quiet, ASR apps performed similarly to listeners with a moderate hearing loss. When transcribing

243 speech-in-noise, the ASR apps performed in the performance range of CI recipients. Sentences-in-

244 noise provided a quick test to assess ASR performance since that test material provided more

245 linguistic cues than digits-in-noise or lists of CNC words.

\section{Performance compared to human listeners}

247 The performance of the ASR apps on speech-in-quiet tests seems comparable to listeners with a

248 moderate conductive hearing loss (30-35 dB threshold shift), which is known as disabling for certain

249 activities in daily life (25). In comparison, Dingemanse \& Goedegebure (2019) found a mean score

250 of $82 \%$ in 50 adult unilateral CI-recipients on the Dutch CNC-test tested in free field at $65 \mathrm{~dB}$ SPL,

251 which is the level of conversational speech (26). This performance may be an overestimation for the

252 average CI-users since they excluded participants with a CNC-score below 60\%. Kaandorp et al.

253 (2015 determined a mean score in free field at $65 \mathrm{~dB}$ SPL of 95\% while using their preferred device

254 in 24 hearing aid users with a moderate to severe hearing loss and $80 \%$ in 24 CI recipients (27). Only 
255 for speech at high-intensity levels, well above the level of conversational speech, do the apps achieve

25690 to $100 \%$ speech reception. The poor performance at low speech intensity levels may be caused by

257 hardware limitations, as discussed below in the section on hardware. The ASR may score lower due

258 to the lack of contextual information provided in the test. The CNC-test was developed as an auditory

259 test that requires little linguistic skill. The listener can only use the consonant-vowel-consonant

260 structure and the fact that the lists contain only familiar existing words. The alternative of using

261 nonsense words, or nonsense sentences, would probably further deteriorate ASR performance while

262 being a valid test for assessing auditory function with a lower effect of language skills by the subject

263 (28). Most ASR are trained on sentences of realistic conversations (8). The strength of (deep

264 learning) ASR is based on using contextual information from a natural language processing model

265 (29). That contextual information is not available in word testing.

266 The performance of the ASR apps on the Digits-in-Noise test was very limited compared to humans.

267 Normal hearing listeners achieve on the DIN-test monaurally using headphones an SNR of $-8.8 \mathrm{~dB}$

268 (23). CI recipients rated on the same criteria as normal hearing listeners, typically achieve DIN scores

269 ranging from +3 to $-6 \mathrm{~dB}$. For instance, Kaandorp et al. (2015) found an average SNR of $-1.8( \pm 2.7)$

$270 \mathrm{~dB}$ in a group of 18 adult unilateral CI recipients in free field test conditions (27). The ASR is at a

271 disadvantage because in the DIN-test, contextual information is lacking and the priors for the ASR

272 and human are not the same. When doing a digits-in-noise test, a human will only report digits. For

273 the ASR it is not a 10-class problem but a problem with several thousand alternatives. The apps tend

274 to construct sentences rather than separate numbers. For conversations where it is important to catch

275 a number, such as the price of an item, the DIN-test might be a useful measure.

276 The performance of the ASR apps on sentences in noise (Plomp-test) was very limited and much

277 poorer than in people with a moderate hearing loss (21). Normal hearing listeners have an SRT at an

278 SNR of -8 to $-10 \mathrm{~dB}(21)$, while the best ASR apps achieved $+8 \mathrm{~dB}$ scores. Kaandorp et al. (2015) 
279 found mean SRT on Dutch Sentences in noise by scoring keywords of $+2.1 \mathrm{~dB}$ for 24 hearing aid

280 users (tested on their preferred ear) with moderate to severe hearing loss and $+8.0 \mathrm{~dB}$ for 24

281 unilateral CI recipients (27). In CI-recipients, evaluation of speech-in-noise is often performed

282 scoring keywords, instead of full sentences as used in the original procedure by Plomp and Mimpen

$283(1979 ; 20)$. In another study, Kaandorp et al (2016) found a significant difference of 1.0 dB in favor

284 of a keyword scoring procedure compared to scoring full sentences (30). However, this $1.0 \mathrm{~dB}$

285 keyword effect does not account for the large difference between the app's performance and the

286 performance of hearing aid users in noise. On the Plomp-test, which provides more linguistic

287 information than the CNC- and DIN-test, the apps' performance is far below that of the majority of

288 hearing impaired listeners and similar to the range of outcomes in CI-recipients.

289 Sentences with and without noise (Plomp-test) could be considered as a performance metric for ASR

290 apps in difficult listening conditions. Possibly with more natural sentences to provide even more

291 linguistic cues. Testing through a loudspeaker has the advantage that it takes the effect of room

292 acoustics into account, making the test condition more realistic. Instead of a sound booth, a room

293 with more representative acoustics for daily situations (e.g the reverberation time of a classroom or

294 using babble noise instead of speech-shaped noise) would provide even more representative results.

295 The current scoring procedure of the Plomp-test, based on fully correct sentences, leads to very high

296 SNRs that may underestimate the practical value of ASR for hearing impaired persons. For instance,

297 if an ASR in a conversation under noisy conditions provides keywords, it may already benefit the

298 person with hearing impairment. One could easily adopt the Plomp-test by determining the WER

299 score on a fixed SNR level to simulate above example. Or alternatively, accept a higher number of

300 mistakes (compared to none) in the adaptive test by using keywords (30). Besides audiological test

301 outcomes, the systematically collected feedback by groups of users (e.g. a focus group) would be 
302 very helpful to further improve the accessibility and usability of ASR apps for hearing impaired

303 listeners.

304 In longer dialogues, all tested apps provided a running English transcript with a WER around 19-

$30534 \%$. This roughly corresponds to $60-80 \%$ correct word ( $\sim 1-\mathrm{WER})$ scores and this is in the same

306 range as for persons with profound hearing loss who use a cochlear implant (31) and better than

307 hearing aid users with a profound hearing loss (32). For these groups, the use of the ASR apps tested

308 here would likely provide benefits.

\section{Hardware and platform variability}

310 A possible explanation for the poor performance at low levels could be the smartphone's microphone

311 gain settings and limited dynamic range rendering soft sounds undetectable (33). We chose a

312 microphone orientation, directing it to the speaker that we assumed was optimal for the task.

313 However, we did not check the directionality of the built-in microphones. In actual use, the

314 microphone orientation could be suboptimal, for instance, if a listener positions the device such that

315 it enables better reading of the transcript from the screen. Also in group settings, the user will likely

316 put the device flat on a table and thus not always point the microphone to the speaker. We did not

317 investigate the effect of suboptimal microphone orientation. Another explanation for the level

318 dependence in quiet could be pre-processing. Most ASR systems usually normalize the input (34).

319 Potentially the ASR systems classify soft sounds as non-speech or not of interest.

320 In English, there is not much difference between the apps or between the operating platforms.

321 Therefore, we do not expect differences in the Dutch version to stem from hardware differences

322 between the smartphones (e.g., microphone sensitivity) but from the implementation of the Dutch

323 language in the specific app or the used training data. The difference between iOS and Android was

324 only visible in Dutch. In Dutch, Earfy (iOS) and Ava (iOS) score significantly poorer. 
325 There was no consistent difference favoring either iOS or Android versions of the apps. Earfy

326 performed better on Android, while AVA performed better on iOS. For prospective users, the

327 performance of the app depends on language, and may depend on the platform.

\section{$328 \quad$ Limitations}

329 The administered tests did not include the effect of accents or speech impairments (e.g. deaf speech;

$330(7,35))$. The displayed transcripts changed during the dialogue, and the transcript was evaluated at the

331 end of the dialogue instead of in real-time. When reading the transcript in real-time, the performance

332 of the speech recognition apps might be better or worse due to the changing words in real-time to

333 construct a logical sentence.

334 When measuring performance in noise, an adaptive SNR procedure was used. The effect of noise

335 could be more extensively studied by evaluating ASR by determining the Word Recognition Score

336 (the convention in the field of audiology) or the Word Error Rate (the convention in the field of ASR

337 research) on several fixed SNR levels (e.g. $-5,0,+5$ and $+10 \mathrm{~dB}$ SNR) that correspond to realistic

338 listening conditions for people using a hearing aid (36). For ecological valid measures, the effect of

339 different fluctuating noise maskers should be considered $(37,38)$. Babble-noise or traffic noise is

340 much more realistic than (artificial) steady-state speech-shaped noise. In the end, the performance of

341 the ASR must be robust enough that users will put their trust in these apps even in formal situations

342 such as a conversation with their doctor or audiologist.

343 In this study, only (audiological) speech-to-text performance of the apps was measured. The

344 usability, processing speed, effect on speechreading, and readability of the transcript were not

345 evaluated. Other researchers looked into requirements for speed and user interface and concluded that

346 those are important factors to improve usability (39). We expect that an increasing number of ASR 
347 apps will adhere to accessibility guidelines to improve usability for the elderly and people with

348 disabilities as promoted by the Web Accessibility Initiative (40).

349 The number of apps tested in this study is limited. We did not perform a standardized procedures for

350 literature review (e.g. PRISMA) to find and include ASR apps for this pilot study. In English, more

351 apps may be available than in Dutch and we did not include expensive state-of-the-art (professional)

352 ASR systems.

353 Other factors to consider not included in this pilot study are the distance between speaker and

354 listener, especially in these times of social distancing and the effect of face masks on a speakers'

355 voice and intelligibility (41). Feedback about voice quality could help the speaker adopt a more

356 intelligible speaker style. The errors made by the ASR may be complementary or redundant to the

357 errors made by persons with hearing loss. We did not study the error patterns. A potential way to

358 determine the complementary effect of ASR could be to evaluate speech-recognition in noise using

359 an audiovisual presentation mode, instead of the audio-only mode that was used in this study, in three

360 distinct aided conditions. 1) participants with hearing loss aided with hearing aid or CI. 2)

361 participants with hearing loss aided with hearing aid or CI and using an ASR app, 3) performance by

362 the ASR app only. Studying the difference between these conditions reveals the added benefit and

363 may penalize ASR systems not designed for simultaneous speechreading and text reading.

365 Metrics to evaluate personalized ASR performance

366 Instead of the quick audiological tests we performed here, a more conventional and elaborate

367 evaluation method would be to record several hours of conversations with hearing impaired users

368 (including realistic lexicon and acoustics) via a smartphone while the screen is oriented such that the 
369 user can read the transcript. Subsequently, one could create transcripts of the recordings by human

370 transcriptions as ground truth, pass the recordings through several ASR apps and determine a

371 performance rating based on WER and other automated metrics such as the semantic distance

372 between the ASR transcript and ground truth (42).

373 ASR may benefit from domain-specific evaluation tools and have domain-specific applications. For

374 instance, Miner et al. (2020) developed a metric based on symptom-focused language in

375 psychotherapy (43). A domain-specific, or even person-specific factor is that prelingually deaf people

376 often have a speech impairment, leading to lower comprehensibility both for normal hearing listeners

377 who are not accustomed to deaf speech and for ASR apps that are not specifically trained on deaf

378 speech. Fortunately, generic ASR models can be used as a pre-trained model that subsequently is

379 trained on a particular task including a-typical speech, accents, or acoustic conditions without

380 incurring the cost of training a full model (44). Recently, researchers from Google started a project,

381 called Parrotron, to create personalized models which could better convert deaf speech than generic

382 ASR systems. WER dropped from $89.2 \%$ for the generic ASR to $32.7 \%$ for the finetuned ASR for a

383 single prelingually deaf subject (35). In addition, the Parrotron system can synthesize the speech of a

384 speech impaired person (i.e. voice conversion) to make the speech sound more natural and

385 comprehensible to the untrained ear.

386 Metrics as, for example, the WER (SNR, RT), or semantic difference (SNR, RT), as functions of

387 signal-to-noise ratio and reverberation time (RT) can provide more ecologically valid estimates of the

388 benefits ASR apps could provide in daily life. Representative SNR values could include $-5,+10,+30$

389 (quiet) dB SNR. For ecological valid measures, realistic fluctuating noise masker should be used

390 (37,38). Reverberation times typically encountered in daily life to consider are $0,0.5$, and 2.5

391 seconds, which corresponds to ideal, classroom (45), and church (46) room acoustics. Presenting the 
392 ASR performance using the WER (SNR, RT) reduces the need to study the characteristic of the

393 corpus on which the ASR was trained and or evaluated.

\section{Future benefits for audiologists}

395 ASR apps can provide benefits in conversations between patients and their audiologists (47). In

396 addition, ASR technology, when further developed, can play a role in computational approaches to

397 audiology (4). For instance, if personalized ASR apps further develop so that atypical speech is better

398 captured, and if ASR achieves normal hearing performance on audiology tests it may provide another

399 use case: patients could perform self-testing (i.e. automated speech audiometry) by repeating the

400 speech they hear to an ASR system trained on their particular voice replacing or enhancing the task

401 of the professional in the audiology center (48). Manual calculation of complex evaluation metrics is

402 not suitable in clinical settings given the excessive time required and may lead to inter-rater

403 variability (49). Automated speech audiometry using algorithms to score performance can be a

404 valuable complement to automated pure-tone threshold audiometry (50). For example, Vinail et al

405 (2016) validated a semi-automatic speech procedure using customized word-lists, in part, provided

406 by the subject to include familiar words. The customized word-lists were recorded with the subject's

407 own voice to incorporate personalized acoustic and articulatory parameters. Speech recognition was

408 evaluated on the customized word-list using an algorithm to determine automatically the number of

409 correctly repeated phonemes. In addition, the use of ASR could open venues to improved

410 (automated) scoring methods in audiology tests. Ratnanather et al (2021) demonstrated how one can

411 automate the alignment of phonemes based on the minimum edit distance between the source speech

412 and the utterances of the subject in real time (51). Visualizing this alignment may provide insights to

413 clinicians about what phonological errors are made. 
414 A factor of variability in rating procedures is that in many speech-in-noise tests, the test is made

415 easier for CI recipients by only scoring correct keywords rather than full sentences $(28,30)$. Although

416 scoring keywords makes the test accessible to a larger population, it reduces the discriminative power

417 between higher- and lower-educated native listeners (30). An ASR could facilitate an automated

418 scoring procedure that differentiates between errors. For instance, using semantic difference between

419 the ASR transcript and ground truth, errors that lead to semantically similar sentences are weighted

420 favorably, leading to a better outcome measure in terms of how well hearing impaired persons can

421 participate in a conversation under adverse circumstances.

\section{CONCLUSION}

424 None of the ASR apps achieved performance close to normal hearing listeners on audiological tests.

425 No app stood out from the others on performance level. On audiological speech tests in quiet, ASR

426 apps performed similarly to listeners with a moderate hearing loss. When transcribing speech-in-

427 noise, the ASR apps performed in the performance range of CI recipients. Sentences-in-noise

428 provided a quick test to assess ASR performance. Additional performance measures are needed to

429 evaluate ASR apps. Besides the speech material also type of noise and the presentation mode audio-

430 only versus audiovisual need to be considered. Adding new performance metrics including the

431 semantic difference as a function of SNR and reverberation time can help to monitor and further

432 improve ASR performance. Clinicians can use benchmarks based on such metrics to counsel

433 prospective users and may benefit from automated procedures. Several hearing impaired listeners,

434 especially CI recipients, report that they benefit from the apps in certain situations(47), which is in

435 accordance with the results of converting a dialogue into text and may stem from complementary 
error patterns of ASR not investigated here. Personalized ASR could increase the number of listeners enjoying the benefits of ASR.

\section{REFERENCES}

1. Saon G, Kurata G, Sercu T, Audhkhasi K, Thomas S, Dimitriadis D, et al. English Conversational Telephone Speech Recognition by Humans and Machines. ArXiv170302136 Cs [Internet]. 2017 Mar 6 [cited 2021 Oct 8]; Available from: http://arxiv.org/abs/1703.02136

2. Xiong W, Droppo J, Huang X, Seide F, Seltzer ML, Stolcke A, et al. Toward Human Parity in Conversational Speech Recognition. IEEEACM Trans Audio Speech Lang Process. 2017 Dec;25(12):2410-23.

3. Kader SE, Eckert AM, Gural-Toth V. Voice-to-Text Technology for Patients with Hearing Loss. Hear J. 2021;74(2):11-4.

4. Wasmann J-WA, Lanting CP, Huinck WJ, Mylanus EAM, van der Laak JWM, Govaerts PJ, et al. Computational Audiology: New Approaches to Advance Hearing Health Care in the Digital Age. Ear Hear. 2021 Dec;42(6):1499-507.

5. Lesica NA, Mehta N, Manjaly JG, Deng L, Wilson BS, Zeng F-G. Harnessing the power of artificial intelligence to transform hearing healthcare and research. Nat Mach Intell. 2021 Oct;3(10):840-9.

6. Jurafsky D, Martin JH. Speech and Language Processing: An Introduction to Natural Language Processing, Computational Linguistics, and Speech Recognition. 2nd ed. Upper Saddle River, N.J.: Pearson Prentice Hall.; 2009.

7. Koenecke A, Nam A, Lake E, Nudell J, Quartey M, Mengesha Z, et al. Racial disparities in automated speech recognition. Proc Natl Acad Sci U S A. 2020 Apr 7;117(14):7684-9.

8. Cieri C, Miller D, Walker K. The Fisher Corpus: a Resource for the Next Generations of Speechto-Text. LREC. 2004;(Vol. 4):69-71.

9. Godfrey JJ, Holliman EC, McDaniel J. SWITCHBOARD: Telephone speech corpus for research and development. In: Acoustics, Speech, and Signal Processing, IEEE International Conference on. IEEE Computer Society; 1992. p. 517-20.

10. Panayotov V, Chen G, Povey D, Khudanpur S. Librispeech: An ASR corpus based on public domain audio books. In: 2015 IEEE International Conference on Acoustics, Speech and Signal Processing (ICASSP). 2015. p. 5206-10. 
11. Kincaid J. Which Automatic Transcription Service is the Most Accurate? - 2018 [Internet]. Descript. 2018 [cited 2021 Oct 1]. Available from: https://medium.com/descript/whichautomatic-transcription-service-is-the-most-accurate-2018-2e859b23ed19

12. Bernstein LE, Tucker PE, Demorest ME. Speech perception without hearing. Percept Psychophys. 2000;62(2):233-52.

13. Helfer KS. Auditory and auditory-visual perception of clear and conversational speech. J Speech

14. Coldewey D. Ava expands its AI captioning to desktop and web apps, and raises $\$ 4.5 \mathrm{M}$ to scale [Internet]. TechCrunch. 2020 [cited 2021 Dec 12]. Available from: https://social.techcrunch.com/2020/12/10/ava-expands-its-ai-captioning-to-desktop-and-webapps-and-raises-4-5m-to-scale/

15. Google. How Google technology is improving accessibility for deaf people - Google [Internet]. About Google. [cited 2021 Dec 11]. Available from: https://about.google/intl/ALL_us/stories/making-conversation-more-accessible-with-livetranscribe

16. Earfy. New Earfy functions based on user feedback! - [Internet]. Earfy. 2017 [cited 2021 Dec 11]. Available from: https://www.earfy.net/earfy/new-functions-for-earfy-based-on-userfeedback/

17. Mattys SL, Davis MH, Bradlow AR, Scott SK. Speech recognition in adverse conditions: A review. Lang Cogn Process. 2012;27(7-8):953-78.

18. Gatehouse S, Naylor G, Elberling C. Benefits from hearing aids in relation to the interaction between the user and the environment. Int J Audiol. 2003 Jan 1;42(sup1):77-85.

19. International Organization for Standardization. ISO 8253-1: 2010. Acoustics: audiometric test methods. Part 1: Pure-tone air and bone conduction audiometry. International Organization for Standardization Geneva; 2010.

20. Bosman AJ, Smoorenburg GF. Intelligibility of Dutch CVC syllables and sentences for listeners with normal hearing and with three types of hearing impairment. Audiology. 1995 Sep 1;34(5):260-84.

21. Plomp R, Mimpen AM. Speech-reception threshold for sentences as a function of age and noise level. J Acoust Soc Am. 1979 Nov;66(5):1333-42.

22. Plomp R, Mimpen AM. Improving the reliability of testing the speech reception threshold for sentences. Audiology. 1979;18(1):43-52.

23. Smits C, Theo Goverts S, Festen JM. The digits-in-noise test: Assessing auditory speech recognition abilities in noise. J Acoust Soc Am. 2013 Mar;133(3):1693-706.

24. Bronkhorst AW, Bosman AJ, Smoorenburg GF. A model for context effects in speech recognition. J Acoust Soc Am. 1993;93(1):499-509. 
25. World Health Organization. World report on hearing [Internet]. World Health Organization; 2021 [cited 2021 Apr 1]. Available from: https://www.who.int/publications-detail-redirect/worldreport-on-hearing

26. Dingemanse JG, Goedegebure A. The Important Role of Contextual Information in Speech Perception in Cochlear Implant Users and Its Consequences in Speech Tests. Trends Hear. 2019 Jan 1;23:2331216519838672.

27. Kaandorp MW, Smits C, Merkus P, Goverts ST, Festen JM. Assessing speech recognition abilities with digits in noise in cochlear implant and hearing aid users. Int J Audiol. 2015 Jan 2;54(1):48-57.

28. O'Neill ER, Parke MN, Kreft HA, Oxenham AJ. Development and validation of sentences without semantic context to complement the basic English lexicon sentences. J Speech Lang Hear Res. 2020;63(11):3847-54.

29. Deng L. Deep learning: from speech recognition to language and multimodal processing. APSIPA Trans Signal Inf Process. 2016;5.

30. Kaandorp MW, De Groot AM, Festen JM, Smits C, Goverts ST. The influence of lexical-access ability and vocabulary knowledge on measures of speech recognition in noise. Int J Audiol. 2016;55(3):157-67.

31. Blamey P, Artieres F, Başkent D, Bergeron F, Beynon A, Burke E, et al. Factors Affecting Auditory Performance of Postlinguistically Deaf Adults Using Cochlear Implants: An Update with 2251 Patients. Audiol Neurotol. 2013;18(1):36-47.

32. Flynn MC, Dowell RC, Clark GM. Aided Speech Recognition Abilities of Adults With a Severe or Severe-to-Profound Hearing Loss. J Speech Lang Hear Res. 1998 Apr;41(2):285-99.

33. Faber BM. Acoustical measurements with smartphones: Possibilities and limitations. Acoust Today. 2017;13(2):10-6.

34. Jakovljević N, Janev M, Pekar D, Mišković D. Energy normalization in automatic speech recognition. In: International Conference on Text, Speech and Dialogue. Springer; 2008. p. 3417.

35. Biadsy F, Weiss RJ, Moreno PJ, Kanevsky D, Jia Y. Parrotron: An end-to-end speech-to-speech conversion model and its applications to hearing-impaired speech and speech separation. ArXiv Prepr ArXiv190404169. 2019;

36. Christensen JH, Saunders GH, Havtorn L, Pontoppidan NH. Real-World Hearing Aid Usage Patterns and Smartphone Connectivity. Front Digit Health. 2021 Aug 20;3:722186.

37. Festen JM, Plomp R. Effects of fluctuating noise and interfering speech on the speech-reception threshold for impaired and normal hearing. J Acoust Soc Am. 1990;88(4):1725-36.

38. Francart T, Van Wieringen A, Wouters J. Comparison of fluctuating maskers for speech recognition tests. Int J Audiol. 2011;50(1):2-13. 
39. Glasser A, Kushalnagar K, Kushalnagar R. Deaf, Hard of Hearing, and Hearing Perspectives on Using Automatic Speech Recognition in Conversation. In: Proceedings of the 19th International ACM SIGACCESS Conference on Computers and Accessibility [Internet]. New York, NY, USA: Association for Computing Machinery; 2017 [cited 2021 Sep 20]. p. 427-32. (ASSETS '17). Available from: https://doi.org/10.1145/3132525.3134781

40. Initiative (WAI) WWA. Home [Internet]. Web Accessibility Initiative (WAI). [cited 2021 Dec 16]. Available from: https://www.w3.org/WAI/

41. Yi H, Pingsterhaus A, Song W. Effects of Wearing Face Masks While Using Different Speaking Styles in Noise on Speech Intelligibility During the COVID-19 Pandemic. Front Psychol. 2021 Jun 28;12:682677.

42. Kim S, Arora A, Le D, Yeh C-F, Fuegen C, Kalinli O, et al. Semantic Distance: A New Metric for ASR Performance Analysis Towards Spoken Language Understanding. ArXiv Prepr ArXiv210402138. 2021;

43. Miner AS, Haque A, Fries JA, Fleming SL, Wilfley DE, Wilson GT, et al. Assessing the accuracy of automatic speech recognition for psychotherapy. NPJ Digit Med. 2020;3(1):1-8.

44. Huang W-C, Hayashi T, Wu Y-C, Kameoka H, Toda T. Voice Transformer Network: Sequenceto-Sequence Voice Conversion Using Transformer with Text-to-Speech Pretraining. ArXiv191206813 Cs Eess [Internet]. 2019 Dec 14 [cited 2021 Dec 11]; Available from: http://arxiv.org/abs/1912.06813

45. Knecht HA, Nelson PB, Whitelaw GM, Feth LL. Background noise levels and reverberation times in unoccupied classrooms. 2002;

46. Desarnaulds V, Carvalho AP, Monay G. Church acoustics and the influence of occupancy. Build Acoust. 2002;9(1):29-47.

47. Berenger M. Hearing Australia [Internet]. New app from National Acoustic Laboratories improves communication at hearing health clinics. [cited 2021 Dec 23]. Available from: https://www.hearing.com.au/About-Hearing-Australia/Hearing-news/New-app-from-NationalAcoustic-Laboratories-improv

48. Venail F, Legris E, Vaerenberg B, Puel J-L, Govaerts PJ, Ceccato JC. Validation of the Frenchlanguage version of the OTOSPEECH automated scoring software package for speech audiometry. Eur Ann Otorhinolaryngol Head Neck Dis. 2016 Apr;133(2):101-6.

49. Smith M, Cunningham KT, Haley KL. Automating error frequency analysis via the phonemic edit distance ratio. J Speech Lang Hear Res. 2019;62(6):1719-23.

50. Wasmann J-W, Pragt L, Eikelboom R, Swanepoel DW. Digital approaches to automated and machine learning assessments of hearing: a scoping review (Preprint). J Med Internet Res [Internet]. 2021 Aug 3 [cited 2021 Dec 17]; Available from: http://preprints.jmir.org/preprint/32581/accepted

51. Ratnanather J, Wang L, Bae S-H, O’Neill E, Sagi E, Tward D. Visualization of Speech Perception Analysis via Phoneme Alignment: A Pilot Study. Front Neurol. 12:724800. 
5787 CONFLICT OF INTEREST

579 The authors declare that the research was conducted in the absence of any commercial or financial

580 relationships that could be construed as a potential conflict of interest.

5818 AUTHOR CONTRIBUTIONS

582 LP, JWW and PvH conceptualized the study. LP, PvH and DG collected the data. All authors

583 contributed to the data interpretation, reviewed the results, and edited the manuscript. LP and JWW

584 took the lead in drafting the manuscript.

5859 FUNDING

586 No funding applicable

$587 \quad 10 \quad$ ACKNOWLEDGMENTS

588 We thank David R. Moore, Paul J. Govaerts, Cas Smits, and J. Tilak Rathanather for their comments

589 on draft versions of the article. From our institution, we thank Cris P. Lanting, Wendy J. Huinck, and

590 Emmanuel A.M. Mylanus for their suggestions over the course of this study. Finally, we thank the

591 reviewers for their comments.

5921 DATA AVAILABILITY STATEMENT

593 The datasets generated for this study can be requested by contacting the corresponding author. 


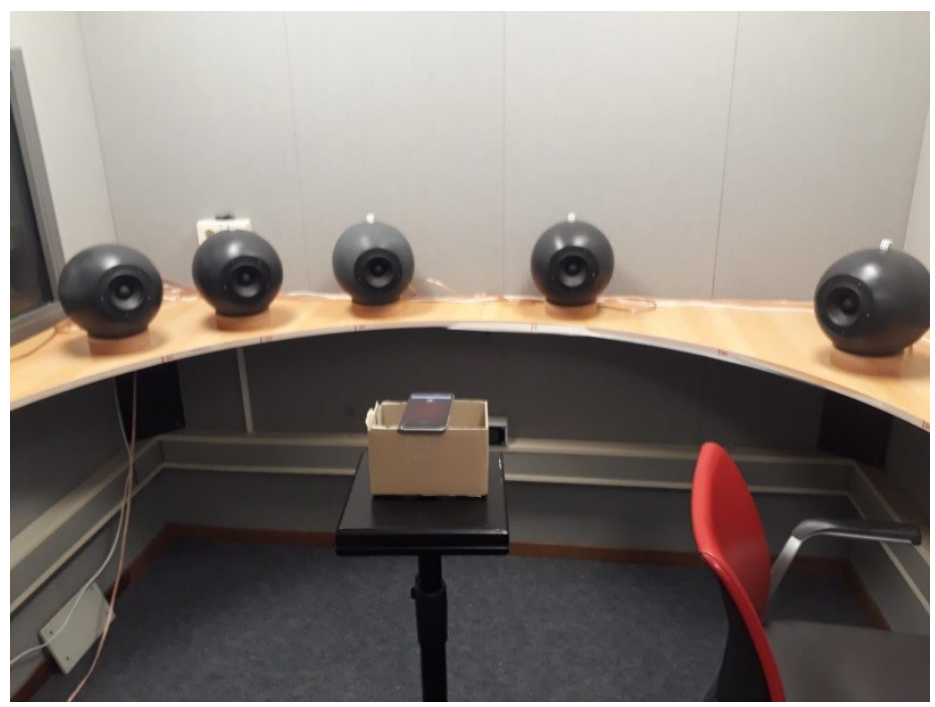

597 Figure 1: Set-up of the smartphone in front of the speaker.

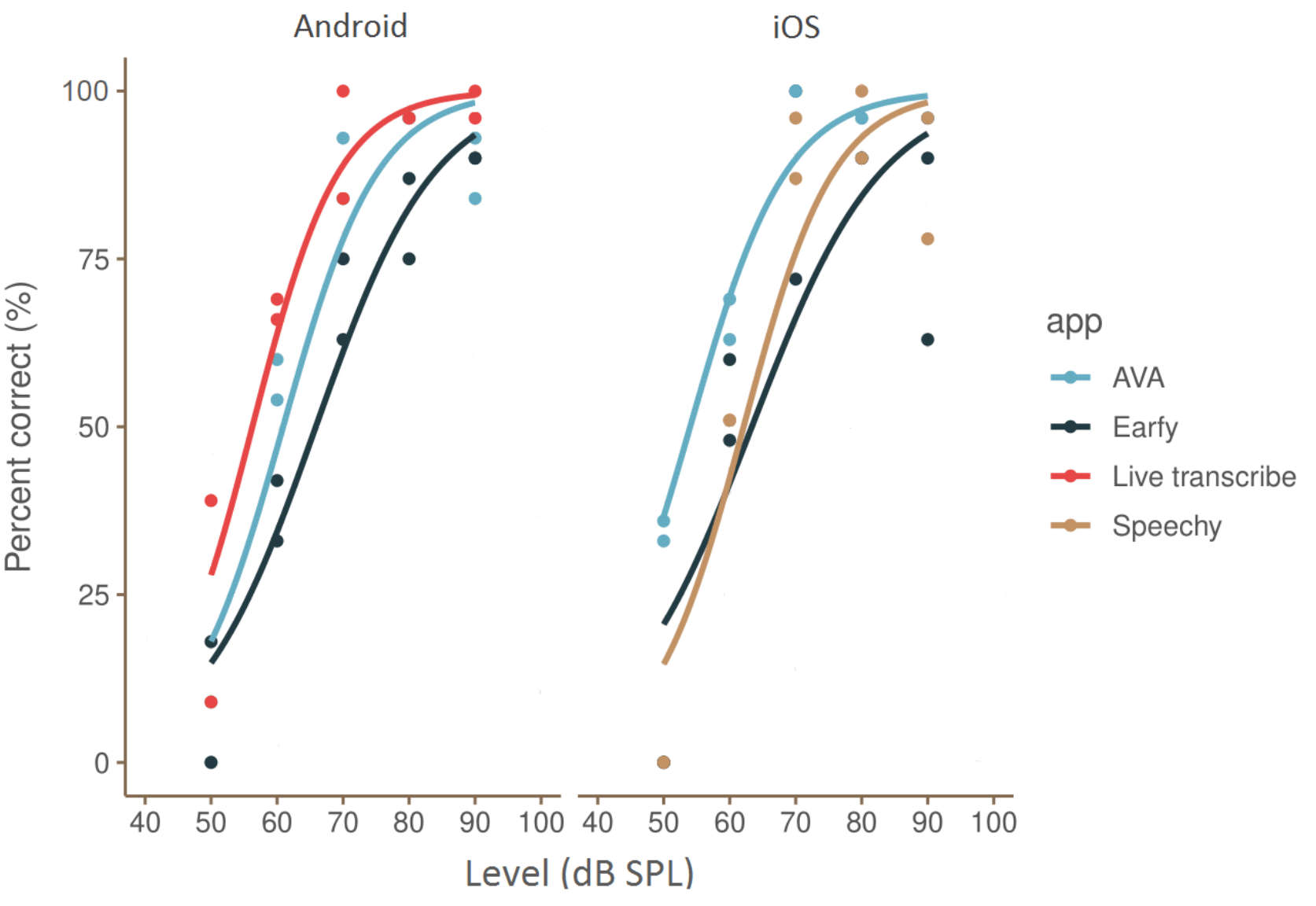

599 Figure 2: Speech recognition as a function of presentation level (in human listeners known as speech 600 audiogram) of all ASR apps tested on an Android and iOS smartphone. The plotted lines are 
601 interpolated using a logistic function through the measured test-retest data-points. Left side: results of 602 the Android apps, right side: results of the iOS apps.

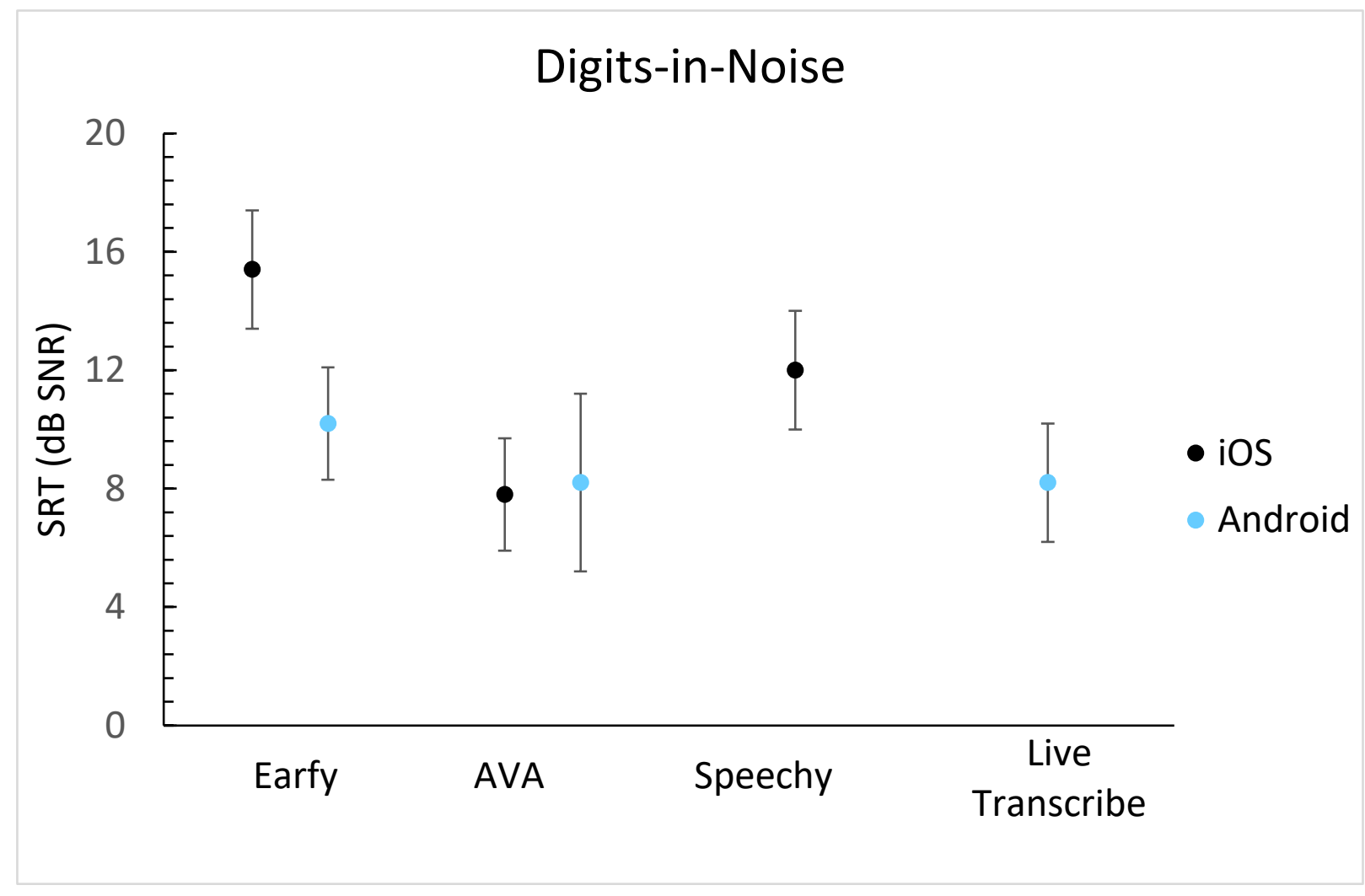

604 Figure 3: Digit in Noise results expressed in SNR per app. A lower score is better. The error bars 605 represent the standard deviation of the response of the app within a single list of triplets. 


\section{Sentences-in-Noise}

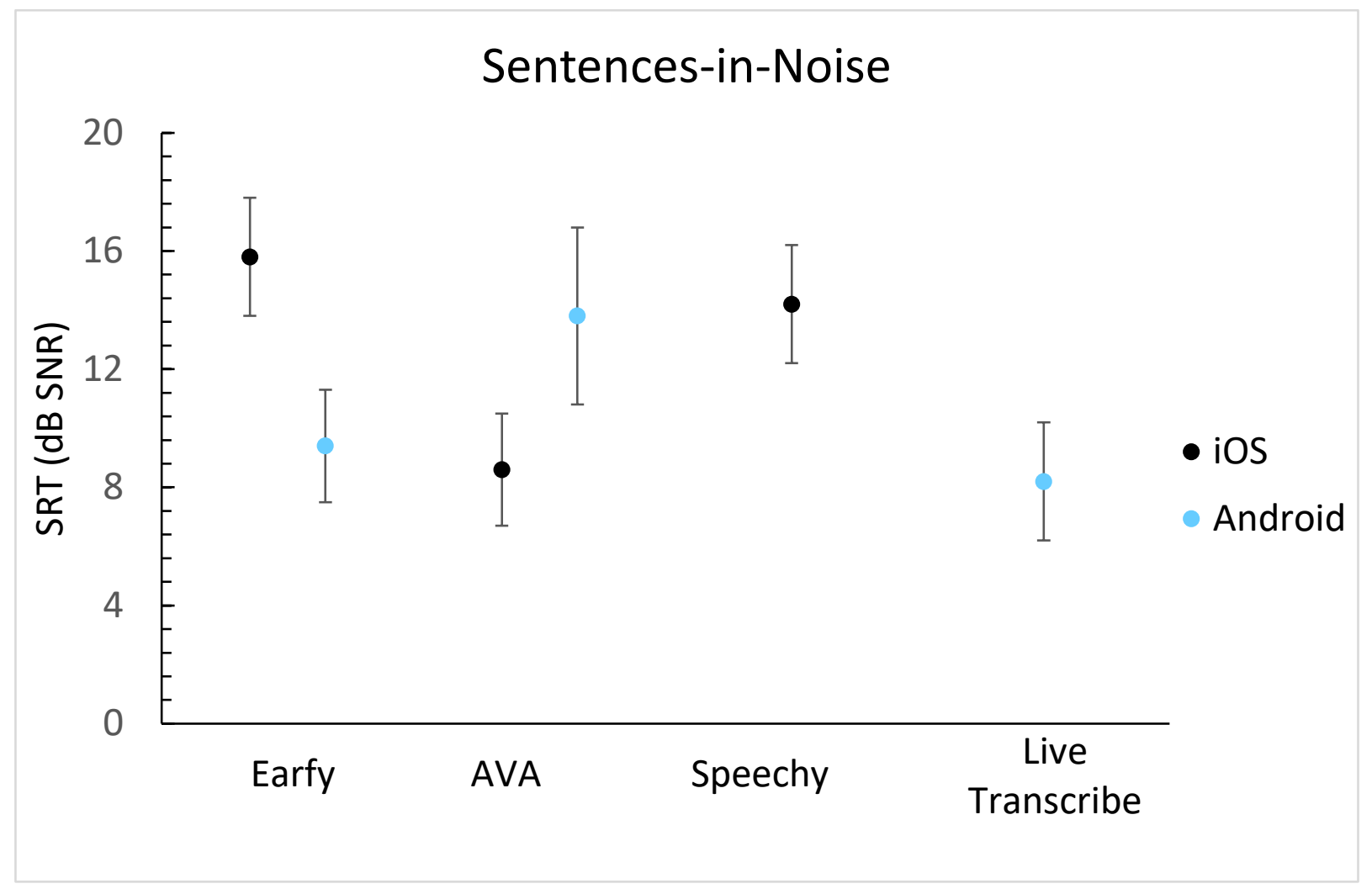

608 Figure 4: Sentences in noise results expressed in SNR per app. A lower score is better. The error bars

609 represent the standard deviation of the response of the app within a single list of sentences. 
automated speech recognition apps for the deaf

\section{Word Error Rate}

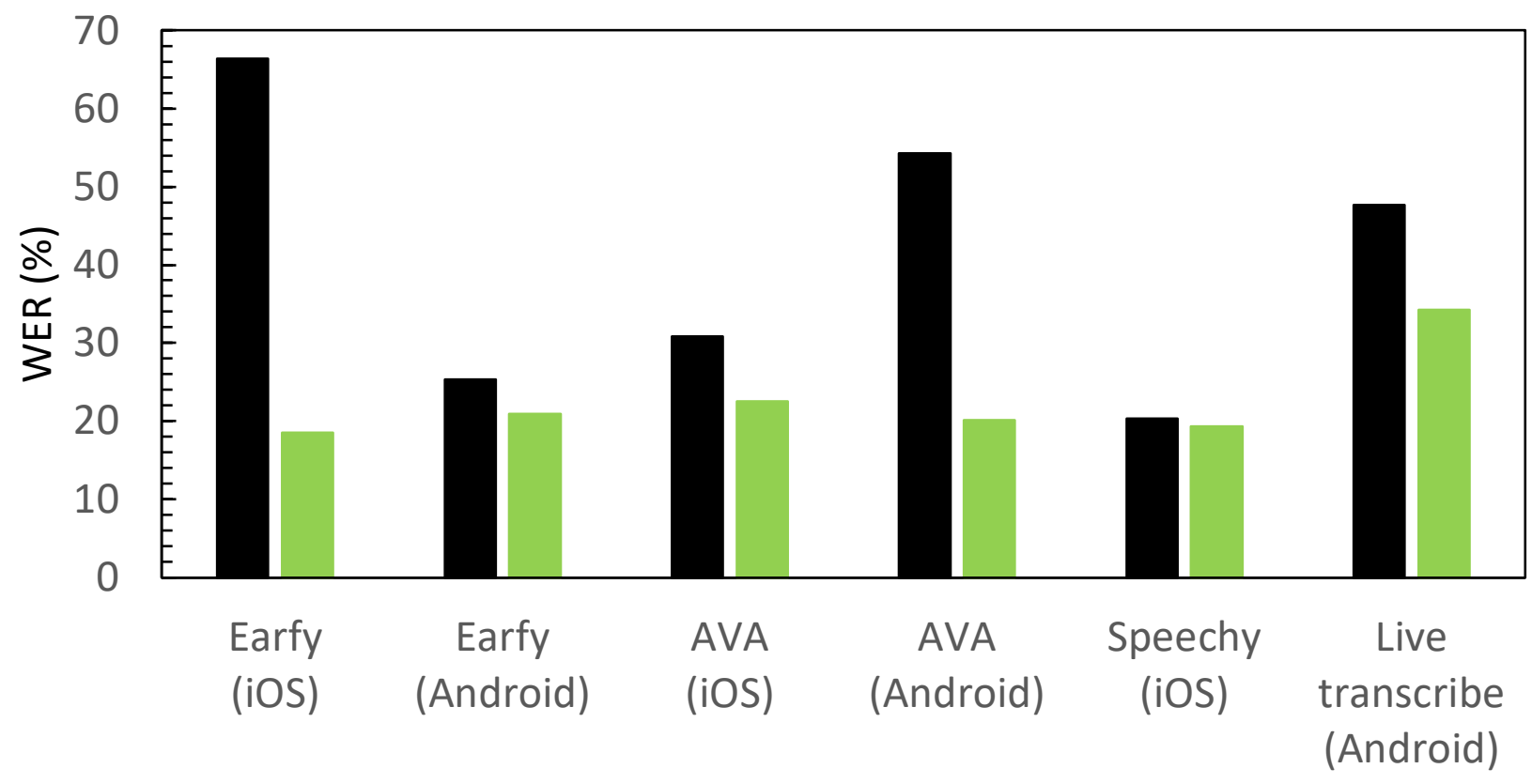

Dutch English

610

611 Figure 5: Word error rate in percentage of the dialogue in English and Dutch for the different apps.

612 


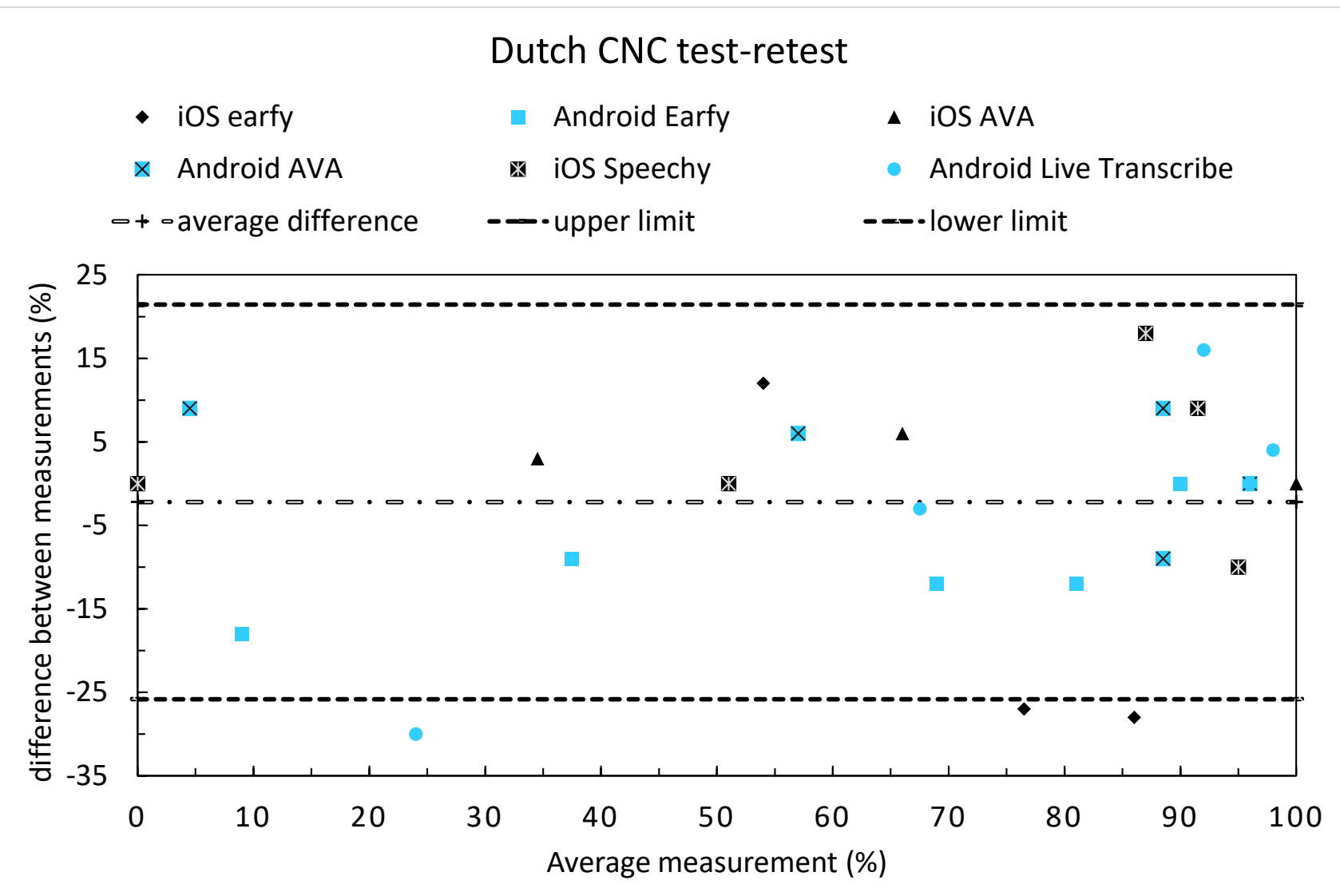

613

614 Figure 6: Bland-Altman plot to display the test-retest reliability of the CNC-test. 